\title{
Segmentation Algorithm of Armor Plate Surface Images Based on Im- proved Visual Attention Mechanism
}

\author{
Yue Chen", Jianhua Zhang and Hongyan Zhang
}

School of Mechatronics Engineering, Xuzhou Institute of Technology, XuZhou, Jiangsu Province, 221111, P.R. China

\begin{abstract}
In the sight of difficulty in segmentation of armor plate surface defects, visual attention mechanism is applied to segment defects images of steel strip surface, the key step of visual attention mechanism-fusion method of saliency images is improved, thinking of the contribution of salient area size, number and distribution to the comprehensive salient image, gray level consistency and GLCM's entropy are used to calculate weight coefficients for feature images fusion, and comprehensive saliency image is obtained, then maximum entropy method is used to segment the comprehensive saliency image. The segmented results are compared to the results of clustering and region-growing methods, the improved visual attention mechanism has better effects.
\end{abstract}

Keywords: Feature fusion, salient feature, segmentation of steel strip surface defect images, visual attention mechanism.

\section{INTRODUCTION}

Defects image segmentation of armor plate surface is the key step of defects detection by visual method, it is the precondition of defects recognition and classification. For the sake of bad working environment, images gotten from rolling site are with low contrast ratio and hard to segment. Generally, there are four segmentation methods: threshholdbased method, edge-based method, region-based method and special theory-based method.

In threshhold-based method, the threshold of gray image pixel value must be confirmed first, then make threshold transform on the image. Those pixels with values bigger than the threshold will be assign a new value, on the other hand, the other pixels will be assign an opposite value. Then the new gotten image has only two pixel values with distinct contrast, this is in favour of ROI extraction.

Edge detection is also an important image segmentation method. It gets RIO of image by extracting common boundary of different region. Different edge detection operators have different segmentation results, such as Canny operator can get an very meticulous boundary, even the weak light reflecting difference in background can also be detected, so the segmentation result can not used in image recognition and classification.

Region-based method include region-growing method and splitting-merging method [1-3]. Region-growing means expanding pixels with similar attributes from one or a few pixels. The region-growing method is sensitive to noise and the seed point in expanding procedure need to be manually determined, it is also time consuming to big target. Splittingmerging method is the opposite process to region-growing method. How to design the splitting and merging criterions is the key factor to get good segmentation result. The algorithm of splitting-merging method is complex and with large calculation, it may also destroy the regions boundary.

Except the above three typical segmentation methods, there are some segmentation methods using special theories. In ensence, each of these methods belongs to one of the above three but has its own theoretical basis such as clustering-based image segmentation [4-7], wavelet-analysis-based image segmentation [8-11], fuzy theory-based image segmentation [12-14], neural network-based image segmentation [15-17], visual attention mechanism based image segmentation [18, 19], etc.

Visual attention mechanism based image segmentation method imitates human visual attention mechanism to focus on the area need to notice, extracts RIO from several saliency maps. Thinking of the low contrast ratio of armor plate surface images and good segmentation results are hard to get by the typical segmentation methods, Visual attention mechanism based image segmention is applied to segment armor plate surface image, also the feature saliency maps fusion method is improved in this paper.

\section{VISUAL ATTENTION MECHANISM}

\subsection{Introduction of Visual Attention Mechanism}

Human can quickly pay attention to those visual objects distinctive from the rest when facing a complex scene, and give priority processing to these objects, this procedure is called Visual Attention, these visual objects are called visual focuses. Important features can be quickly attentained and processed by Visual Attention mechanism but the features commonly possesed with other things are been turned a blind eye to them, this is called selective attention mechanism [20]. Generally, Visual Attention mechanism includes three parts: visual features, visual saliency and focus shift. Visual 
features includes color, brightness, direction features and motion feature for moving objects. Visual saliency is the measurement of the differences between objects and envirnment, it is also the underlying cause that lead to visual attention, to measure the visual saliency is the key step to realize visual attention model. For measuring visual saliency, saliency features must be extracted-a saliency image equal to original image in size. There are two saliency features: self saliency features(extracted from objects themselves) and mutual saliency features(extracted from outside region). A visual scene always contains several attentional focuses, so the viewpoint shifts from one focus to anther when one is observing it.

Visual attention mechanism model is divided into two types: down-top(what) and top- down(where). The down-top model is an unsupervised model, the watcher has no prior knowledge and focus eyes on objects according to the real situation;In the top-down model, the watcher focuses eyes on objects in accordance with prior knowledge.

Itti visual attention mechanism $[21,22]$ is based on frame of neurobiology put forward by Koch and Ulman. Itti model proposes a down-top mechanism and feature images can be computed parallelly, it posts a better performance in visual inspection and computing speed. By lineral filtering, color, brightness and orientation feature maps can be extracted;Then by center-arround operator and normalization, each feature map can be acquired;Finally, comprehensive saliency map can be acquired by feature fusion. The comprehensive saliency map can discribe the incitement degree of different regions to eyes of an image and quantify the saliency of different regions of the saliency map, the saliency regions are confirmed by the values of saliency.

\subsection{Selection of Saliency Features}

The salient region's stimulation to eyes is the key factor in selecting saliency features. Generally, the following features may come into notice: brightness, orientation, contrast to peripheries, local complexity, rareness, local information entropy, color and so on. In this paper, images to be processed are of the armor plate surfaces, the color feature is not obvious, and color images are time consuming in processing and occupying much storage space. So images from worksite are converted into gray images to save processing time and storage space. Local complexity feature is similar to local information entropy, so brightness, rareness and local complexity features are selected to generate saliency map. But there are limitations of each feature in creating saliency map, as a supplement, DoG bandpass spatial filter is used to generate full resolution saliency map, then each feature saliency map and full resolution map are fused to create the comprehensive saliency map.

\subsubsection{Brightness of Pixels}

Take the average of all pixels in a gray image as reference, each pixel value subtract from the reference to get the $\mathrm{D}$-value, then the D-value is processed to get saliency map. The formulas is as following:

$$
I(x, y)=|f(x, y)-\bar{f}|
$$

$$
S_{I}(x, y)=1-\exp \left(-\frac{I(x, y)}{\sum_{x^{\prime}=1}^{M} \sum_{y^{\prime}=1}^{N} I\left(x^{\prime}, y^{\prime}\right)}\right)
$$

$f(x, y)$ is the pixel value of $(x, y)$, the image size is $\mathrm{M} \times \mathrm{N}$. $S_{\mathrm{I}}(x, y)$ is brightness saliency map. D-value is normalized and amplified by formula (2).

\subsubsection{Rareness Feature}

Rareness means the distinction of a pixel from the rest, more different from the rest pixels, much information contained and saliency more apparent. Generally, pixels in background have high percentage of all pixels with little information, so they have little significance; On the other hand, some defect regions have relatively few pixels but more distinctive from the background and more salient. So rareness can be used to discribe the saliency of regions in an image, the formula is as following:

$$
S_{R}(x, y)=\frac{1}{\operatorname{hist}(f(x, y))}
$$

$\operatorname{hist}(\cdot)$ is the histogram of the image, $S_{\mathrm{R}}(x, y)$ is the rareness saliency value of pixel $f(x, y)$.

\subsubsection{Local Complexity}

Local complexity is to discribe the pixels gray value changes of regions in an image. MSE (mean squared error) and RPVC (rate of pixels values change) are used to discribe local complexity.

RPVC:

$$
C_{p}=\frac{k}{2 M N-M-N}
$$

$$
\text { MSE: }
$$

$$
\sigma=\sqrt{\frac{1}{M N} \sum_{i=1}^{M} \sum_{j=1}^{N}(f(i, j)-\bar{f})^{2}}
$$

$\mathrm{M} \times \mathrm{N}$ is the size of a region in an image, $\bar{f}$ is the average of all the pixels values in the region, $\mathrm{k}$ is the number neighbour pixels that values change.

(1) If $C_{\mathrm{p}} \geq T_{\mathrm{C}}, \sigma \geq T_{\sigma}$, then there are many neighbour pixels with changed gray values and the range of variation is larger, this region can be considered more salient.

(2) If $C_{\mathrm{p}} \leq T_{\mathrm{C}}, \sigma \geq T_{\sigma}$, then there are little neighbour pixels with changed gray values, but the range of variation is larger, there maybe mutational site in the region.

(3) If $C_{\mathrm{p}} \leq T_{\mathrm{C}}, \sigma \leq T_{\sigma}$, then there are little neighbour pixels with changed gray values and the range of variation is smaller, this region is belong to background, the pixels values change is the fluctuation.

(4) If $C_{\mathrm{p}} \geq T_{\mathrm{C}}, \sigma \leq T_{\sigma}$, then there are many neighbour pixels with changed gray values, but the range of variation is smaller, this region is belong to background too. 


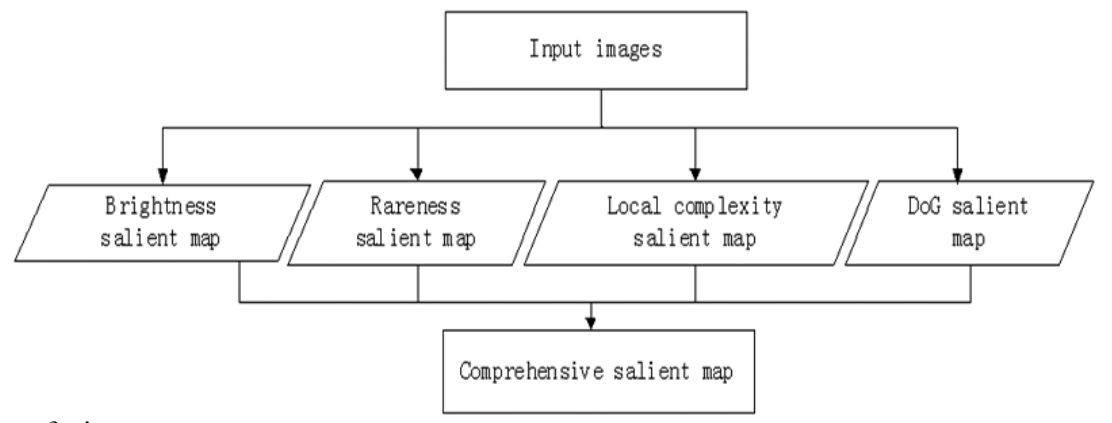

Fig. (1). Schematic of feature fusion.

From the above analysis we know that MSE is the key factor in pixels saliency judgement, RPVC can not give the change range of pixels values variation. Take $\sigma$ to calculate saliency:

$$
S_{C}=1-\exp \left(-\frac{|f(i, j)-\bar{f}|}{2 \sigma^{2}}\right)
$$

The greater the pixels values' deviation from the mean, the more salient the pixels are.

\subsubsection{DoG spatial Band-Pass Filtering}

DoG(Difference of Gaussian) and LoG(Laplacian of Gaussian) are similar to each other. DoG can be used to enhance the edges and other details to be visible.2-D DoG defined as following:

$$
\begin{aligned}
& \operatorname{DoG}(x, y)=\frac{1}{2 \pi}\left[\frac{1}{\sigma_{1}^{2}} e^{-\frac{\left(x^{2}+y^{2}\right)}{2 \sigma_{1}^{2}}}-\frac{1}{\sigma_{2}^{2}} e^{-\frac{\left(x^{2}+y^{2}\right)}{2 \sigma_{2}^{2}}}\right] \\
& =G\left(x, y, \sigma_{1}\right)-G\left(x, y, \sigma_{2}\right)
\end{aligned}
$$

DoG is similar to Mexican hat wavelet by subtraction of two Gaussian fuctions. The ratio of two Gaussian fuctions standard deviations- $\sigma_{1}: \sigma_{2}$-are used to control the width of passing band, if $\sigma_{1}: \sigma_{2}=1.6$, DoG can closely approximates LoG as an ideal edge detecter; if $\sigma_{1}: \sigma_{2}=5$, DoG can closely approximates retina nerve cells' view. So pixels can be filtered by controlling the value of $\sigma_{1}: \sigma_{2}$, if $\sigma_{1}: \sigma_{2}=\infty, \mathrm{DC}$ component(pixels of background) is filtered out. For filtering out high frequency noises and thin textures, Gaussian kernel$[1,4,6,4,1] / 16$-is always used to approximates Gaussian function.

A salient map of an image can be obtained by formula(7) after being processed by DoG.

$S_{G}(x, y)=\left|I_{\mu}-I_{G}(x, y)\right|$

$I_{\mu}$ is the average of inputing image pixels values, $I_{\mathrm{G}}(x, y)$ is the image after being processed by Gaussian filter.

\subsection{Fusion of Salient Maps}

Each salient map acquired from above four features demonstrated saliency of only one feature, so each of them has limitation-salient region in one salient map but not salient in another one. Therefore, the four salient maps should be fused together to acquire a comprhensive salient map to segment the original image, as shown in the Fig. (1).

The weight of each salient map determined as follows [23]: Detecting salient points, this can be done by extracting pixels with values bigger than a salient map's threshold, then computering fusion weight of each salient map by the number of salient points, position of salient points and distribution of salient points. Three weights can be computered from these three factors, by these three weights, the fusion weight of each salient map in acquiring the comprehensive salient map can be obtained.

$$
W_{i}=W_{S p}+W_{L}+W_{D}
$$

$W_{\mathrm{i}}$ is the final weight of one salient map in features fusion, the comprehensive salient map can be acquired by formula (8):

$S=\sum_{i=1}^{n} W_{i} S_{i}$

$S_{\mathrm{i}}$ is a feature salient map. This fusion method confirms to human visual system, but it is difficult to determin the threshold in extracting salient region. Further more, if there are multiple salient regions, the subsequent calculation of distances between salient regions is large and the distribution of salient regions can not be expressed. In view of the effectiveness of complexity in discribling the area and distribution of salient regions, image omplexity and entropy are used to computer the feature fusion weights.

The size, number and distribution of salient regions in a salient map are the factors determing the role of the salient map in generating the comprehensive salient map. Pixels consistency complexity can express the uniformity of an image pixels, GLCM entropy can expresses the randomness of textures. The salient regions are more dispersive, the entropy is more bigger and vise versa. So using pixels consistency and GLCM entropy to computer the fusion weights is feasible.

Let $U=\left[U_{1}, U_{2}, \ldots, U_{4}\right]$ expresses feature salient maps pixels consistency complexities of an image, then each fusion weight of feature salient map is:

$w_{U i}=\frac{U_{i}}{\max (U)} \quad i=1,2,3,4$

Let $H=\left[H_{1}, H_{2}, \ldots H_{4}\right]$ expresses feature salient maps GLCM entropies of an image, then each fusion weight of feature salient map is: 


$$
w_{H i}=\frac{H_{i}}{\max (H)} \quad i=1,2,3,4
$$

The comprehensive weight of each feature salient map is:

$$
w_{i}=w_{H i}+w_{U i}
$$

The comprehensive salient map can be obtained by the following formula:

$$
S=\sum_{i} w_{i} S_{i}{ }_{i=1,2,3,4}
$$

\section{SEGMENTATION OF DEFECTS OF ARMOR PLATE IMAGES BASED ON VISUAL ATTENTION MECHANISM}

\subsection{Generation of Feature Salient Maps}

Take the above method to generate feature salient maps of images in Fig. (2). First the original images should be rectified to make the images brightness even, avoiding the influence of bright speck on generating feature salient maps.

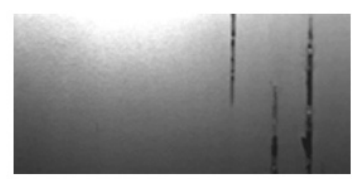

(a) Shells

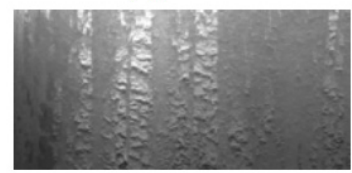

(c) Scales

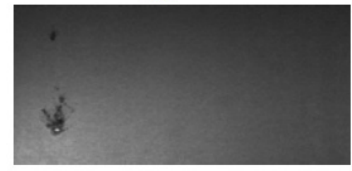

(e) Rolled in dirt

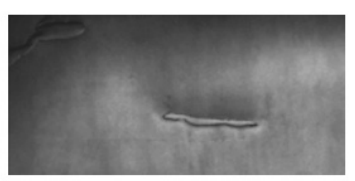

(b) Spalls

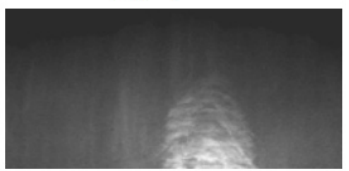

(d) Bruise

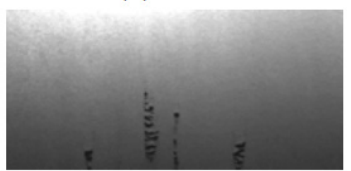

(f) Sagger
Fig. (2). Samples of original defect images.

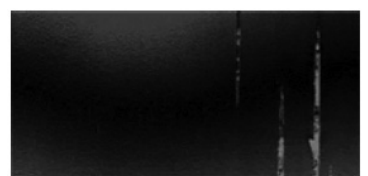

(a) Shells

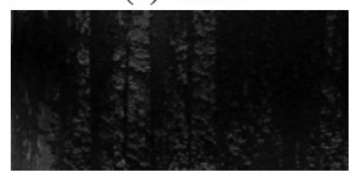

(c) Scales

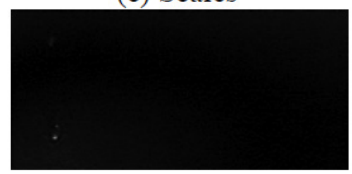

(e) Rolled in dirt

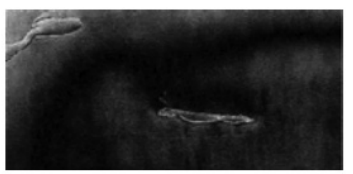

(b) Spalls

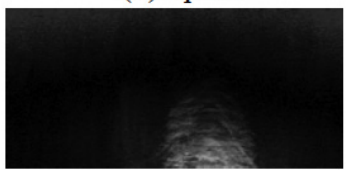

(d) Bruise

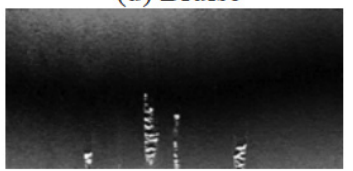

(f) Sagger
Fig. (3). Samples of brightness salient images.

\subsubsection{Brightness Salient Maps}

Fig. (3) shows the brightness salient maps of images in Fig. (2) obtained by the above method.

From Fig. (3) we can see that the brightness salient map can display the defects regions in images, but the uneven backgrounds have bad effect on salient maps although they were rectified before.

\subsubsection{Pixels Rareness Salient Maps}

As showed in Fig. (4), salient regions are not obvious in rareness salient maps, this is because most pixels in defect regions are not different from each other, although there are distinctive in vision, but in histogram, only pixels in edges are distinctive from other pixels.

\subsubsection{Local Complexity Salient Maps}

In order not to miss pixels on images boundaries, images are expanded by adding pixels rows and colums around images, just copy the boundaries pixels. Then take $3 \times 3$ pixels region to calculate local complexity, the salient map is as Fig. (5).

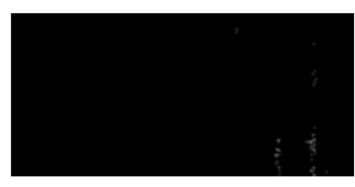

(a) shells

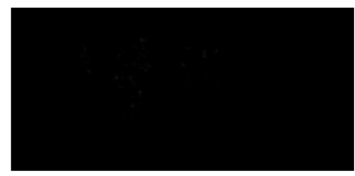

(c) scales

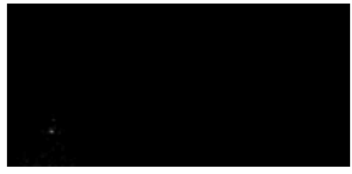

(e) Rolled in dirt

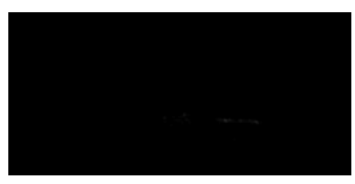

(b) spalls

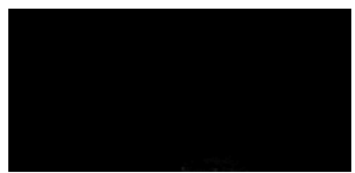

(d) bruise

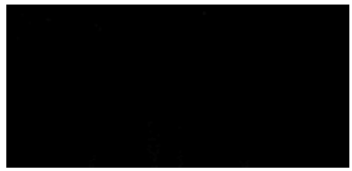

(f) Sagger
Fig. (4). Samples of rareness salient images.

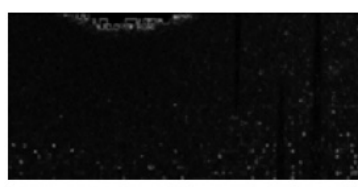

(a) Shells

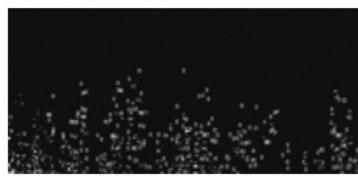

(c) Scales

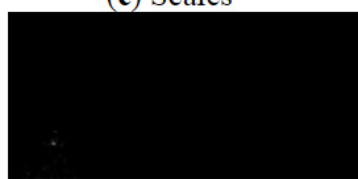

(e) Rolled in dirt

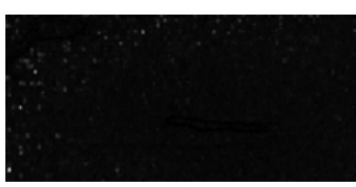

(b) Spalls

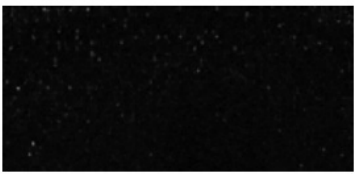

(d) bruise

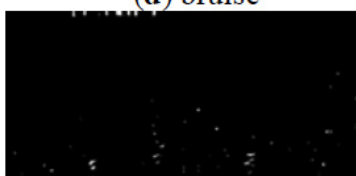

(f) Sagger
Fig. (5). Samples of local complexity salient images. 
The fluctuation of background pixels has effect on local complexity extracting for pixels in defect regions are similar to those in background, so the salient regions in salient maps are the pixels with obvious fluctuation, and defect regions are not salient in salient maps, that is, local complexity salient maps have little dedication in generating comprehensive salient map.

\subsubsection{DoG Filtering Salient Map}

By experimentation, we take $\sigma_{1}: \sigma_{2}=5: 1, \sigma_{1}=0.5, \sigma_{2}=0.1$ to calculate the DoG filtering salient maps, the salient map is as Fig. (6).

In DoG filtering salient maps, obvious defect regions correspond obvious salient regions, but for weak defects or defects with fuzzy edges, they are not salient enough.

\subsection{Fusion of Feature Salient Maps}

Feature salient maps obtained above should be fused together to get comprehensive salient map, different feature salient map makes different contribution to comprehensive salient map. The equal weights fusion method can not express these difference. In view of the number and distribution of salient regions in salient maps, Pixels consistency and GLCM entropy are used as weights to fused feature salient maps. the results calculated are as following Tables 1-5:

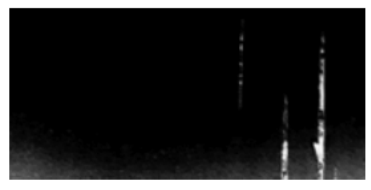

(a) Shells

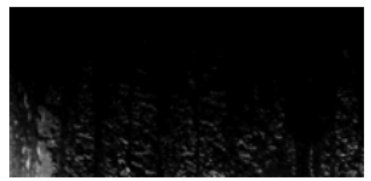

(c) Scales

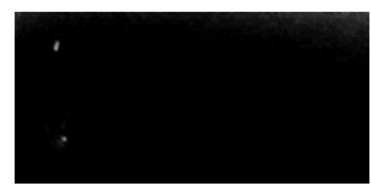

(e) Rolled in dirt

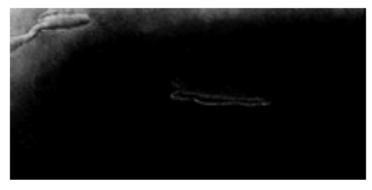

(b) Spalls

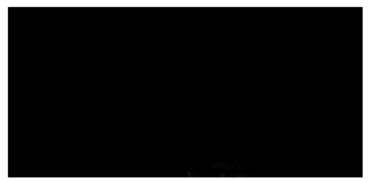

(d) Bruise

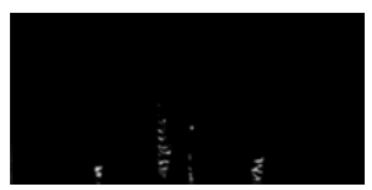

(f) Sagger
Fig. (6). Samples of DoG salient images.

By formula (9) and (10), weights of salient maps are obtained as following tables:

The comprehensive salient map obtained by fusing feature salient maps is as Fig. (7).

Table 1. Gray consistency values of feature salient images.

\begin{tabular}{|c|c|c|c|c|c|}
\hline & Shell & Spall & Scale & Bruise & Dirt \\
\hline \hline Brightness & 503.292 & 839.4828 & 1009.7 & 505.69 \\
\hline Rareness & 453.985 & 454.82 & 454.47 & 461.9583 & 444.34 \\
\hline Complexity & 2431.3 & 762.7615 & 3729 & 560.0533 & 722.43 \\
\hline DoG & 851.37 & 1046.4 & 1589.2 & 739.21 & 332.51 \\
\hline
\end{tabular}

Table 2. GLCM entropy values of feature salient images.

\begin{tabular}{|c|c|c|c|c|c|c|}
\hline & Shell & Spall & Scale & Bruise & Dirt & Sagger \\
\hline Brightness & 2.2892 & 2.7360 & 2.3313 & 1.5741 & 2.0273 & 2.5482 \\
\hline Rareness & 0.7049 & 0.7116 & 0.7090 & 0.7128 & 0.7055 & 0.7094 \\
\hline Complexity & 2.8672 & 1.6860 & 1.3489 & 1.3275 & 1.6122 & 2.5935 \\
\hline DoG & 1.2715 & 1.3202 & 1.2309 & 0.7726 & 1.4951 & 1.4764 \\
\hline
\end{tabular}

Table 3. Weight coefficients of gray consistency.

\begin{tabular}{|c|c|c|c|c|c|c|}
\hline & Shell & Spall & Scale & Bruise & Dirt & Sagger \\
\hline Brightness & 0.207 & 0.8023 & 0.3079 & 0.6841 & 0.6151 & 0.2738 \\
\hline Rareness & 0.1867 & 0.4347 & 0.1386 & 0.6249 & 0.6244 & 0.2781 \\
\hline Complexity & 1 & 0.7289 & 1 & 0.7576 & 1 & 1 \\
\hline DoG & 0.3502 & 1 & 0.4846 & 1 & 0.4603 & 0.5468 \\
\hline
\end{tabular}


Table 4. Weight coefficients of GLCM entropy.

\begin{tabular}{|c|c|c|c|c|c|c|}
\hline & Shell & Spall & Scale & Bruise & Dirt & 1 \\
\hline \hline Brightness & 0.7984 & 1 & 1 & 1 & 0.9825 \\
\hline Rareness & 0.2458 & 0.2601 & 0.3041 & 0.4528 & 0.3480 & 0.2735 \\
\hline Complexity & 1 & 0.6162 & 0.5786 & 0.8433 & 0.7952 & 0.7375 \\
\hline DoG & 0.4435 & 0.4825 & 0.5279 & 0.4908 & 0.5693 \\
\hline
\end{tabular}

Table 5. Comprehensive weight coefficients of feature salients.

\begin{tabular}{|c|c|c|c|c|c|c|}
\hline & Shell & Spall & Scale & Bruise & Dirt & 1.6151 \\
\hline \hline Brightness & 0.9964 & 1.8023 & 1.3079 & 1.6841 & 1.2563 \\
\hline Rareness & 0.4325 & 0.6984 & 0.4427 & 1.0777 & 0.9724 & 0.5516 \\
\hline Complexity & 2 & 1.3451 & 1.5786 & 1.6009 & 1.7952 & 1.1978 \\
\hline DoG & 0.7937 & 1.4825 & 1.0125 & 1.4908 & 1.1431 \\
\hline
\end{tabular}

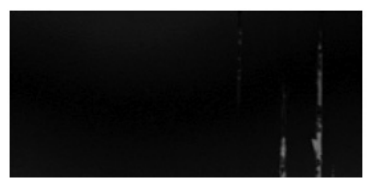

(a) Shells

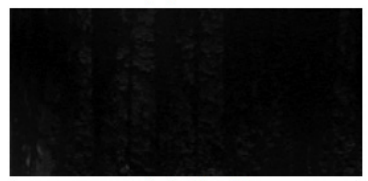

(c) Scales

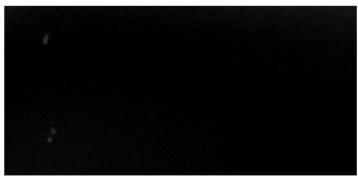

(e) Rolled in dirt

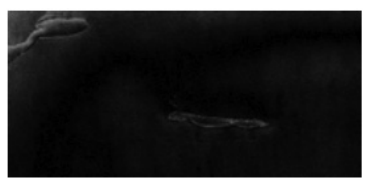

(b) Spalls

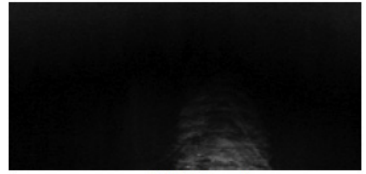

(d) Bruise

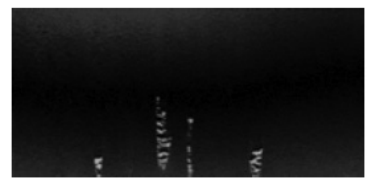

(f) Sagger
Fig. (7). Samples of comprehensive salient images.

\subsection{Defect Regions Segmentation of Comprehensive Sali- ent Maps Based on Maximum Information Entropy}

\subsubsection{Maximum Information Entropy}

In order to segment defect regions, salient regions in comprehensive salient map should be seprated from background. We use maximum information entropy to segment comprehensive salient maps.

The maximum information entropy principle refers to taking the threshold corresponding to maximum information entropy of image as segmentation threshold. The formula is:

$E_{n}=-\sum_{i=1}^{L} p_{i} \ln \left(p_{i}\right)$

$p_{\mathrm{i}}$ is the probability of the occurrence of pixels with value $i . L$ is the range of value $i$. When the pixels values distribute equally, that is, $p_{\mathrm{i}}=1 / \mathrm{N}(\mathrm{N}$ is the number of pixels $)$, maximum information entropy is obtained. In actual images, pixels distributing equally is impossible. In this case, if taking a threshold to seprate forground from background, then calculate the information entropies of forground and background, the pixels number of forground and background reduces and the the variation range is smaller. If the forground and background can be accurately separated by the threshold, the sum of information entropies is maximum.

\subsubsection{Determing the Threshold}

The comprehensive salient map includes the salient regions attracting visual attention. Salient region segmentation can be realized by discretizing and binaryzing comprehensive salient map to get salient region and non salient region. There will be error in the segmented region because the salient regions may not correspond to the defect regions in images, especially edges and isolated salient points. So morphological operator should be used to erose the isolated points, then eliminate the empty regions by expansion operation to obtain complete target regions. The threshold is obtained by the following formulas:

$$
E_{B}=\frac{-\sum_{i=1}^{t} p_{i}}{\sum_{j=1}^{t} p_{j}} \times \ln \frac{p_{i}}{\sum_{j=1}^{t} p_{j}} E_{F}=\frac{-\sum_{i=t+1}^{L} p_{i}}{1-\sum_{j=1}^{t} p_{j}} \times \ln \frac{p_{i}}{1-\sum_{j=1}^{t} p_{j}}
$$

$T=\arg _{t} \max \left(E_{F}+E_{B}\right)$

$E_{\mathrm{B}}$ is the information entropy of background, $E_{\mathrm{F}}$ is the information entropy of forground. $L$ is the maximum pixel value in comprehensive salient map. $p_{\mathrm{i}}$ is the the probability of pixels appearance with value $i$. Pixels with values greater than threshold are set 1 , those smaller than threshold are set 0 , then the binary image is obtained. 


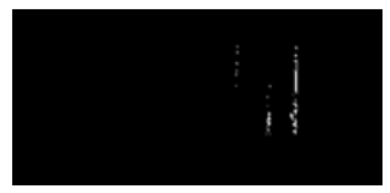

(a) Shells

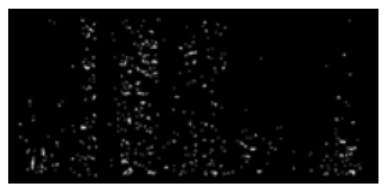

(c) Scales

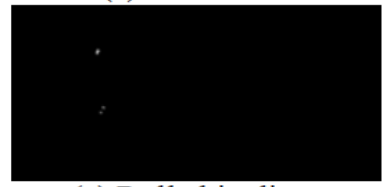

(e) Rolled in dirt

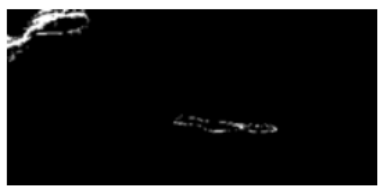

(b) Spalls

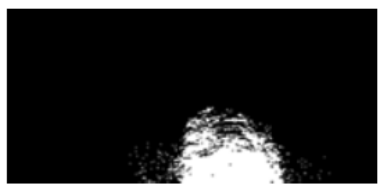

(d) Bruise

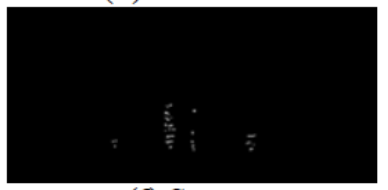

(f) Sagger

Fig. (8). Segmented results of maximum information entropy.

\subsubsection{The Results of Segmentation}

Fig. (8) is the results of segmentation by maximum information entropy method.

\section{THE COMPARATION OF SEGMENTATION RE- SULTS}

K-means clustering segmentation method and regiongrowing segmentation method are taken to segment the same images and the results are compared to the segmentation results obtained from the above method. The results of these two segmentation method are as Figs. (9) and (10).

In the segmentation results of K-means, defect regions can be segmented from original images, but the defect regions are not continuous with little pixels blocks. If defects are not distinctive from background, defects can not segmented from orinigal images. that is to say, K-means is not very effective in segmenting images with weak contrast and complex thin textures.

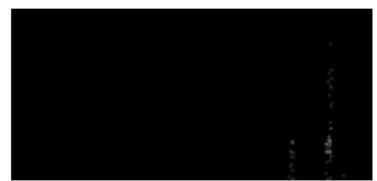

(a) Shells

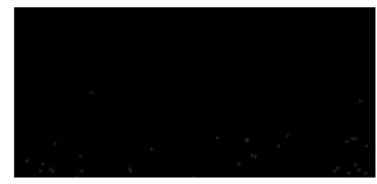

(c) Scales

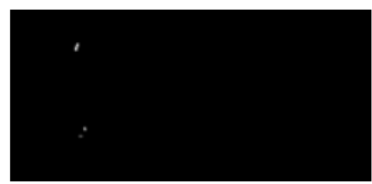

(e) Rolled in dirt

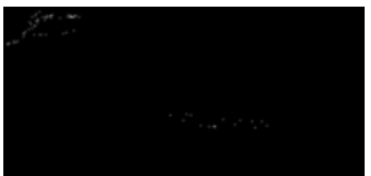

(b) Spalls

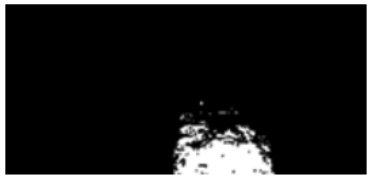

(d) Bruise

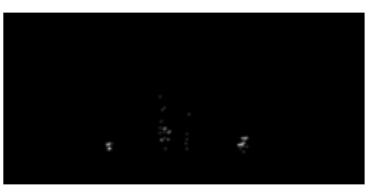

(f) Sagger

Fig. (9). K-means clustering segmentation results.

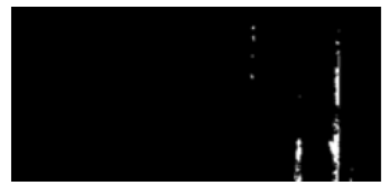

(a) Shells

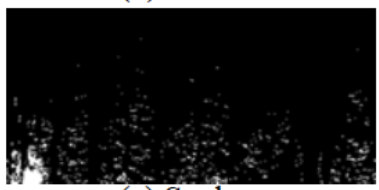

(c) Scales

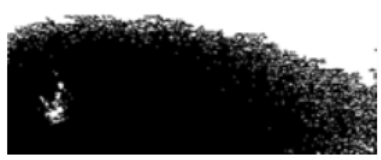

(e) Rolled in dirt

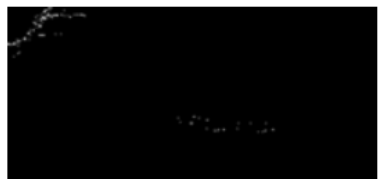

(b) Spalls

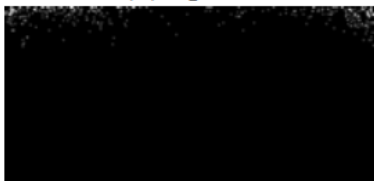

(d) Bruise

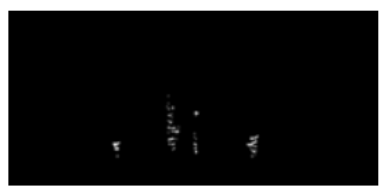

(f) Sagger
Fig. (10). Region-growing segmented results.

Region-growing segmentation method is effective in segmenting images with obvious contrast, if the edges is not clear, such as bruise and rolled-in-dirt defects, the segmentation results are not good enough, even the defects can not be segmented from orinigal images. Any more, for different defects, the seed point and threshold are different too, these will have a great influence on the segmentation results. Comparatively, the segmentation method used in this paper is valuable in segmenting different armor plate surface images.

\section{CONCLUSION}

Visual attention mechanism are improved in image segmentations. In early feature selection, brightness, rareness and local complexity are taken as features to generate feature salient maps by analysis of defect images. Then these feature salient maps are fused together to obtain comprehensive salient maps. Normalized complexities and entropies of feature salient maps are taken as weights in the fusion. This can embody the contribution of each feature salient map to the comprehensive map. On the basis of comprehensive salient maps, maximum information entropy segmentation method is used to segment comprehensive salient maps to get the defect regions, generally, the salient regions in comprehensive maps are defect regions.

In actual armor plate surface defect images segmentation, the results of segmentation have some punctiform non-defect regions if the original images have many thin textures, this is because the high brightness of these thin textures badly influence the comprehensive salient maps. By further filtering this influence can be decreased but can not removed. So the segmentation method should be further researched.

\section{CONFLICT OF INTEREST}

The authors confirm that this article content has no conflict of interest.

\section{ACKNOWLEDGEMENTS}

The authors would express their appreciation for the financial support of Jiangsu High Educational fundamental 
research, grant NO.10KJD510010. The authors also would express their thanks to Yibiao Mao from Shagang Group who offered the armor plate images to this paper.

\section{REFERENCES}

[1] P. Yu, A. K. Qin, and D. A. Clausi, "Unsupervised polarimetric SAR image segmentation and classification using region growing with edge penalty," Geoscience and Remote Sensing, vol. 50, no. 4, pp. 1302-1317, 2012.

[2] J. C. Tilton, Y. Tarabalka, P. M. Montesano, and E. Gofman, "Best merge region-growing segmentationwith integrated nonadjacent region object aggregation," Geoscience and Remote Sensing, vol. 50, no. 11, pp. 4454-4467, 2012.

[3] L. Song, Y. Lv, and B. Yang, "Segmentation of breast masses using adaptiveregion growing," In: $8^{\text {th }}$ International Forum on Strategic Technology, vol. 2, pp. 77-81, 2013.

[4] Y. Hong, Q. Duan, and D. Li, "An improved -means clustering algorithm for fish image segmentation," Mathematical and Computer Modelling, vol. 58, no. 34, pp. 790-798, 2013.

[5] P. R. Bai, Q. Y. Liu, L. Li, S. H. Teng, J. Li, and M. Y. Cao, "A novel region-based level set method initialized with mean shift clustering for automated medical image segmentation, " Computers in Biology and Medicine, vol. 43, no. 11, pp. 1827-1832, 2013.

[6] Z. Wang, Q. Song, Y. C. Soh, and K. Sim, "An adaptive spatial information-theoretic fuzzy clustering algorithm for image segmentation, " Computer Vision and Image Understanding, vol. 117, no. 10, pp. 1412-1420, 2013.

[7] H. Liu, F. Zhao, and L. Jiao, "Fuzzy spectral clustering with robust spatial information for image segmentation," Applied Soft Computing, vol. 12, no. 11, pp. 3636-3647, 2012.

[8] H. Choi, and R.G. Baraniuk, "Multiscale image segmentation using wavelet-domain hidden Markov models," Image Processing, vol. 10, no. 9, pp. 1309-1321, 2001.

[9] A. Dima, M. Scholz, K. Obermayer, "Automatic segmentation and skeletonization of neurons from confocal microscopy images based on the 3-D wavelet transform," Image Processing, vol. 11, no. 7, pp. 790-801, 2002.

[10] M. Acharyya, and M.K. Kundu, "Document image segmentation using wavelet scale-space features," Circuits and Systems for Video Technology, vol. 12, no. 12, pp. 1117-1127, 2002.
[11] S. Kumar, R. Gupta, and N. Khanna, "Text extraction and document image segmentationusing matched wavelets and MRF model, "Image Processing, vol. 16, no. 8, pp. 2117-2128, 2007.

[12] O. J. Tobias, and R. Seara, "Image segmentation by histogram thresholding using fuzzy sets, " Image Processing, vol. 11, no. 12, pp. 1457-1465, 2002.

[13] J.K. Udupa, P. K. Saha, R. A. Lotufo, "Disclaimer: Relative fuzzy connectedness and object definition: theory, algorithms, and applications in image segmentation, "Pattern Analysis and Machine Intelligence, vol. 24, no. 11, pp. 1494-1500, 2002.

[14] V. Boskovitz, and H. Guterman, "An adaptive neuro-fuzzy system for automaticimage segmentation and edge detection," Fuzzy Systems, vol. 10, no. 2, pp. 247-262, 2002.

[15] S. Tao, M.M. Jamshidi, and R.R. Lee, "A modified probabilistic neural network for partial volume segmentation in brain MR image," Neural Networks, vol. 18, no. 5, pp. 1424-1432, 2007.

[16] M. Awad, K. Chehdi, and A. Nasri, "Multicomponent image segmentation using a genetic algorithm and artificial neural network," Geoscience and Remote Sensing Letters, vol. 4, no. 4, pp. 571-575, 2007.

[17] H. L. Zhuang, K.S. Low, W.Y. Yau, "Multichannel pulse coupled neural network based color image segmentation for object detection," Industrial Electronics, vol. 59, no. 8, pp. 3299-3308, 2012.

[18] L. Gao, F. Bi, and J. Yang, "Visual attention based model for target detection in large-field images," Systems Engineering and Electronics, vol. 22, no. 1, pp. 150-156, 2011.

[19] M.C.Q. Farias, W. Y. L. Akamine, "On performance of image quality metrics enhanced with visual attention computational models, ”Electronics Letters, vol. 48, no. 11, pp. 631-633, 2012.

[20] J. Cong, and Y. Yan, "Application of human visual attention mechanism in surface defect inspection of steel strip," China Mechanical Engineering, vol. 22, no. 10, pp. 1189-1192, 2011.

[21] L. Itti, "Automatic foveation for video compression using a neurobiological model of visual attention," Image processing, vol. 13, no. 10, pp. 1304-1318, 2004.

[22] L. Itti, Models of Bottom-up and Top-down visual Attention, USA, California Institute of Technology, 2000.

[23] Q. Zhang, J. Li, H. Xiao, and H. Liu, "Image segmentation based on visual saliency, "Journal of Image and Graphics, vol. 16, no. 5, pp. 767-772, 2011.

(C) Chen et al.; Licensee Bentham Open.

This is an open access article licensed under the terms of the Creative Commons Attribution Non-Commercial License (http: //creativecommons.org/licenses/by-nc/3.0/) which permits unrestricted, non-commercial use, distribution and reproduction in any medium, provided the work is properly cited. 